\title{
Grain-Scale Measurement of Slip Resistances in Aluminum Polycrystals using Spherical Nanoindentation
}

\author{
Shraddha J. Vachhani ${ }^{1,2}$ and Surya R. Kalidindi ${ }^{1 *}$ \\ ${ }^{1}$ Woodruff School of Mechanical Engineering, Georgia Institute of Technology, Atlanta, GA \\ 30332, USA \\ ${ }^{2}$ Currently at Materials Science and Technology Division, Los Alamos National Laboratories, \\ Los Alamos, NM 87545, USA \\ *corresponding author
}

\begin{abstract}
In this work, we develop and demonstrate novel protocols based on spherical nanoindentation and orientation image mapping (OIM) for quantifying the local increases in slip resistances in the individual grains of a deformed (or strain hardened) polycrystalline sample. These new protocols utilize the recently developed data analyses methods for extracting indentation stress-strain (ISS) curves in conjunction with the measurements of the local crystal orientations at the indentation sites using the OIM technique. The proposed protocols involve two main steps. In the first step, spherical nanoindentation measurements are conducted on fully annealed samples of the material of interest to map out the functional dependence of the indentation yield strength $\left(Y_{\text {ind }}\right)$ on the crystal lattice orientation in the annealed condition. In the second step, spherical nanoindentation and OIM measurements are conducted on the deformed samples of the same material and are analyzed rigorously to reliably estimate the increase in the local slip resistance at the indentation sites. The function established in the first step is utilized in the second step to properly account for the influence of the local crystal orientation on the measured $Y_{\text {ind }}$ in the deformed sample. This novel measurement and data analysis protocol is demonstrated in this paper on polycrystalline samples of high purity aluminum. From this study, it was noted that the influence of the crystal lattice orientation on the measured $Y_{\text {ind }}$ in $\mathrm{Al}$ crystals can be as high as $40 \%$, with the lowest values corresponding to the [100] (cube) orientation and highest values corresponding to the [111] orientation. The measurements on the deformed samples showed a significant variation in the strain hardening rates in the individual grains of the polycrystalline sample. A positive correlation was observed between the percentage increase in the local slip resistance and the value of the Taylor factor computed for the local crystal orientation at the indentation site subjected to the macroscale imposed deformation.
\end{abstract}

Keywords: Spherical Nanoindentation, Orientation Imaging, Indentation Yield, Taylor Factor, Indentation Stress-Strain Curves 


\section{Introduction}

Over the last decade, nanoindentation [1-4] has emerged as a tool of choice for investigating mechanical behavior of hierarchical materials, since the interrogation volume under the indenter can be varied systematically by roughly three orders of magnitude in length scales in the range of $50 \mathrm{nms}$ to $50 \mu \mathrm{ms}$. While the indentation experiments are easy to perform and require minimal sample preparation (compared to other small scale mechanical testing options such as micropillar testing [5-7]]), the data analysis and interpretation is quite complicated, mainly due to the complex continuously evolving stress state under the indenter tip. Traditionally, indentation experiments have been carried out with sharp tips [8-10], and the values of local elastic modulus and hardness were extracted mainly from an analysis of the unloading portion of the test segment [11-13]. However, recent advances in instrumentation (e.g., the availability of the continuous stiffness measurement (CSM) [14]) have now made it possible to convert the measured loaddisplacement data from spherical nanoindentation into highly reproducible and consistent indentation stress-strain (ISS) curves [15]. Examining the indentation data in the form of ISS curves provides better insights into the local mechanical response in the sample (although it still needs to be interpreted as an average over the indentation zone experiencing highly heterogeneous stress/strain fields). More specifically, it was demonstrated that these new protocols produce meaningful information such as the local indentation modulus and the local indentation yield strength $\left(Y_{\text {ind }}\right)$. In recent work, the use of ISS curves for nanoindentation data analysis has demonstrated tremendous promise in providing new insights into material behavior, including the role of grain boundaries during macroscale deformation in metals [16], buckling behavior of carbon nanotube forests [17], and lamellar level properties in bone [18].

In this work, we build on prior work from our research group that focused on the characterization of the changes in the local slip resistance in deformed polycrystalline samples of cubic metals [19]. The main concept underlying these new protocols was already introduced in our earlier paper, and involved the utilization and combined analysis of measured data from both spherical nanoindentation and orientation imaging microscopy (OIM) [20-21]. This particular analysis protocol was prompted by the recognition that the measured $Y_{\text {ind }}$ in deformed samples is influenced by both the local crystal lattice orientation and the local dislocation density in a multiplicative manner (details presented later). In order to isolate the contribution from the local dislocation density, it becomes essential to establish a protocol that correctly accounts for the effect of the crystal lattice orientation at the indentation site. Consequently, in our prior work, we establish a new protocol that approached the task in two distinct steps. In the first step, the focus was placed on the annealed samples of the material system of interest (even though our interest is really in the deformed samples), where there is negligible dislocation density in the samples. In this way, one can map out the functional dependence of the $Y_{\text {ind }}$ on the local crystal orientation in the indentation zone. Subsequently, in the second step of the protocols, the focus is shifted to the deformed samples of actual interest. However, the information gathered from the first step (on the annealed samples) is critically important in relating the measured $Y_{\text {ind }}$ to the local percentage 
increase in the slip resistance at the indentation site. It should be noted that this protocol provides a single value for the effective (or the averaged) slip resistance at the indentation site and is therefore most easily interpreted for cubic metals, where it is reasonable to assume that the slip resistance of the different slip systems at the material point of interest are roughly equal to each other. Extension of this protocol to hexagonal metals (with a multitude of slip and twin systems with large variations in their corresponding slip and twin resistances even at a single material point) needs a more detailed analysis.

Our prior work [19] explored these new concepts on polycrystalline Fe-Si samples that exhibit a body-centered cubic (bcc) structure. Although the earlier work established a strong foundation, much additional development is needed to improve the fidelity and robustness of these novel protocols. In this study, we have undertaken a much more extensive effort with the following salient distinctions from the earlier work: (i) The present study was performed on polycrystalline samples of high purity Aluminum (Al), which exhibits a face-centered cubic (fcc) structure. The main reasons for the selection of Aluminum was the relatively simple set of welldefined slips systems (the bcc metals exhibit pencil glide where multiple planes possessing the (111) directions could serve as potential slip systems) and very low elastic anisotropy. Both of these factors are expected to simplify significantly the subsequent analyses of the data presented here. (ii) The samples selected for the present study have been much more systematically processed, corresponding to 0,10 , and 20 percent reductions in plane strain compression. In the earlier work, the samples corresponded to 0,30 , and 80 percent reductions, which made it very difficult to correlate the increases in slip resistances to the amount of cold work imposed on the sample. (iii) The number of measurements performed within a single orientation as well as the number of orientations tested in both annealed and deformed conditions are both substantially larger in this study. Consequently, the present study allowed for a much more meaningful statistical investigation of the correlations sought in the study. (iv) A number of the steps involved in the protocols have been refined to increase the reliability and robustness of the method. For example, it was discovered that the use of a $100 \square \mathrm{m}$ radius indenter tip in the measurements on the annealed sample helped minimize the uncertainty introduced into the analyses as a consequence of the unavoidable pop-ins in these tests. In a similar vein, the protocols for extracting the $Y_{\text {ind }}$ and for capturing mathematically the functional dependence of the $Y_{\text {ind }}$ on the crystal lattice orientation were both substantially refined in the present study.

The overall study presented here is aimed at gaining quantitative insights into the strain hardening rates in the individual grains of a polycrystalline sample. These measurements are critical for maturing physics-based crystal plasticity models [22-25]. Towards this goal, we present our measurements from the study and our analyses of these results. The measurements are provided as tables in this paper to allow other researchers to conduct their own analyses of the main experimental results obtained here using different assumptions, approximations, and crystal plasticity models. 


\section{Indentation Stress-Strain (ISS) Curves}

The procedures used in this study to convert the raw load-displacement data in spherical nanoindentation into ISS curves have been detailed in prior publications $[15,26]$, and are briefly reviewed here. The procedure, based on Hertz theory [27-28], involves the accurate determination of the effective zero point and the computation of the indentation stress and strain values. The effective point of initial contact (may or may not correspond to the actual point of contact) [15] is identified such that the initial elastic loading data segment immediately following this point provides the best agreement with the Hertz theory for all three measured signals: the load $(\tilde{P})$, the displacement $(\tilde{h})$, and the contact stiffness $(S)$. For spherical nanoindentation, this search for the effective initial point can be accomplished using the following relationship based on Hertz theory:

$$
S=\frac{3 P}{2 h_{e}}=\frac{3\left(\tilde{P}-P^{*}\right)}{\left(\tilde{h}-h^{*}\right)}
$$

where $P^{*}$ and $h^{*}$ denote the load and displacement values respectively at the point of initial contact. Linear regression can be used to determine the effective point of initial contact or the socalled effective zero-point (i.e., the values of $P^{*}$ and $h^{*}$ ).

The radius of contact $(a)$ can be estimated as

$$
\begin{aligned}
& a=\frac{S}{2 E_{\text {eff }}}, \\
& \frac{1}{E_{\text {eff }}}=\frac{1-v_{s}^{2}}{E_{s}}+\frac{1-v_{i}^{2}}{E_{i}}
\end{aligned}
$$

where $S$ is the harmonic contact stiffness. $E_{\text {eff }}$ is the effective stiffness of the sample-indenter system, $E$ and $v$ are the Young's modulus and Poisson's ratio, and the subscripts $i$ and $s$ denote the indenter and sample, respectively. The term $\frac{E_{s}}{1-v_{s}^{2}}$ is generally referred to as the sample indentation modulus, denoted by $E_{\text {ind }}$. The value of $E_{\text {ind }}$ is estimated from an analyses of the initial loading segment (identified in the zero-point analyses described above) using the Hertz theory. The values of the indentation stress $\left(\sigma_{\text {ind }}\right)$ and the indentation strain $\left(\varepsilon_{\text {ind }}\right)$ for the initial elastic loading segment are then computed as

$$
\sigma_{\text {ind }}=\frac{P}{\pi a^{2}}, \quad \varepsilon_{\text {ind }}=\frac{4}{3 \pi} \frac{h_{e}}{a} \approx \frac{h_{e}}{2.4 a}, \quad \sigma_{\text {ind }}=E_{\text {ind }} \varepsilon_{\text {ind }},
$$

where $h_{e}$ is the elastic indentation depth at load $P$. The use of Eq. (3) produces a linear relationship between the indentation stress and indentation strain values for the initial elastic loading regime. Beyond this initial elastic regime, the definition of indentation stress is kept the 
same, but the definition of the indentation strain is extended by replacing the elastic indentation depth $\left(h_{e}\right)$ by the total indentation depth $\left(h_{t}\right)$ in Eq. (3). Even in this post-elastic regime, every unloading segment of the CSM is elastic. Hence the Hertz equations are still applicable to the unloading segments of the superimposed CSM oscillations and Eq. (2) still provides an accurate estimate of $a$ even after any amount of plastic deformation [16, 26, 29].

Note that the protocols described above rely on the CSM to obtain a continuous estimate of the radius of contact, $a$, at every point on the load-displacement curve. Studies have shown that the use of CSM is most reliable within certain windows of frequency and amplitude for the superimposed oscillations [30-31]. It has been shown [31] that the most reliable (ISS) curves for aluminum can be obtained using the CSM at $45 \mathrm{~Hz}$ oscillation frequency and a $2 \mathrm{~nm}$ oscillation amplitude. These test conditions were utilized in all of the measurements reported in this paper.

\section{Materials and Methods}

Polycrystalline samples of high purity aluminum (99.999\%) were used in this study. Since our interest here is mainly on the hardening rates within grains (not on the grain boundary regions) we produced fully annealed, large grained, samples of aluminum for this study. This was accomplished by sectioning out samples from a heavily rolled block of high purity aluminum and annealing them for 72 hours at $640^{\circ} \mathrm{C}$. These large-grained samples make it easier to validate the novel protocols being developed in this effort by allowing several measurements within selected grains (of specific orientations) without being influenced by the grain boundaries. As mentioned earlier, the annealed samples are critical for establishing the functional dependence of the $Y_{\text {ind }}$ on the crystal lattice orientation. Some of the annealed samples were subsequently deformed in plane strain compression to two reduction levels of $10 \%$ and $20 \%$. These reduction levels correspond to true strains of -0.11 , and -0.22 , respectively. The deformed samples were studied using the protocols described in this paper to quantify the increases in slip resistances in the grains of different lattice orientations.

As mentioned earlier, one of the reasons for choosing pure aluminum was that it exhibits very little elastic anisotropy $(A=1.22)$ [32]. This is desirable, since the data analysis protocols for nanoindentation are based on Hertz theory, which is based on a frictionless contact between two elastically isotropic surfaces. A modified form of Hertz theory [33]does exist to account for elastic anisotropy in cubic crystals. This theory predicts the sample indentation modulus for Al grains of different orientations to be in the range of 68.8 - 70.6 GPa. Therefore, there should be very little effect of the crystal orientation on the sample indentation modulus in the present study. Additionally, the oxide film on aluminum is exceedingly thin, making its contribution to the indentation response negligible. Absence of solute also eliminates complications due to segregation and precipitation during plastic deformation.

For both the indentation and OIM measurements, the samples were mounted in epoxy and prepared by mechanical grinding using Si-C papers followed by polishing using the $9 \mu \mathrm{m}, 3 \mu \mathrm{m}$ and $1 \mu \mathrm{m}$ diamond suspensions on a Buehler grinding and polishing machine. Mechanical polishing was followed by electropolishing using a mixture of perchloric acid (10\%) and 
methanol for 45 seconds at $-20^{\circ} \mathrm{C}$ and $12 \mathrm{~V}$ [34]. It is emphasized that a high quality surface finish is extremely critical for the indentation measurements [35], especially since we intend to extract mechanical properties from the very small initial elastic loading segments.

Grain orientation maps for the samples were obtained using orientation imaging microscopy (OIM), which is based on the automatic indexing of electron back-scattered diffraction (EBSD) patterns. Orientation at each measurement voxel was denoted using the Bunge-Euler angles [36], as an ordered set of three angles $\left(\varphi_{1}, \Phi, \varphi_{2}\right)$, which describe the rotations for the transformation from the sample reference frame to the crystal reference frame. The grains tested on the sample surfaces were numbered for convenience and will be referred to using their assigned grain numbers. The grain maps were used to identify regions of interest to perform the indentation testing. After the indentation tests were performed, the regions of interest were imaged again in the SEM using OIM, to confirm the location of the indents and determine the local crystal orientation at the indentation site.

Nanoindentation tests were then carried out at selected locations in the annealed and deformed samples using the MTS nanoindenter XP® equipped with the CSM attachment. Care was taken to ensure that the indents were placed well away from the grain boundaries. Twenty seven grains of different orientations, spread out over the inverse pole figure (IPF) triangle were identified on the surfaces of the fully annealed samples for the indentation measurements. Between 9 and 18 indentation tests were performed on each of the selected grains using the protocols outlined in the previous section, and the (ISS) curves were plotted for each test. In the case of the deformed samples, at least 15 indents were performed in each grain due to the increased heterogeneity in the microstructure of deformed samples and the higher expected scatter within each grain.

As reported in prior studies [35] indentation measurements performed on annealed metal samples using a small radius indenter tip almost always produce pop-ins. In fact, this observation was utilized in this study to ensure that the surface preparation during the polishing steps left an undisturbed surface on the sample (i.e., the surface is still representative of the original sample and has not been tainted by the polishing steps). In other words, the efficacy of the surface preparation protocols on the annealed samples used in this study was verified by ensuring that large pop-ins were produced when indents were carried out with the $1 \mu \mathrm{m}$ indenter tip (see Figure 1). These pop-ins are attributed to the difficulty of establishing a potent dislocation source (e.g., a Frank-Reed source) in the very small indentation zone sizes $(<<1 \mu \mathrm{m})$ associated with these measurements.

The main challenge with using the smaller indenter tips (desired because they can lead to higher spatial resolution of the indentation measurements) is that the pop-ins manifest themselves as strain bursts at increased stresses in the ISS curves followed by an unloading part before the indentation stresses approach values that represent indentation flow stresses in the absence of the pop-in [35, 37]. Thus, the occurrence of pop-ins masks the yield behavior and the extraction of $Y_{\text {ind }}$ becomes somewhat unreliable. The effect of indenter tip size on the occurrence 
and size of the pop-ins is shown in Figure 1. Since the indentation zone is much larger for a larger indenter tip, the probability of establishing the necessary dislocation sources to accommodate the imposed deformation is significantly higher, and hence the probability of the occurrence of the pop-in is greatly reduced. Because of these advantages, it was decided to conduct the measurements in this study using the $100 \mu \mathrm{m}$ radius indenter tip.

Even with the $100 \mu \mathrm{m}$ radius indenter tip, small pop-ins were occasionally observed in the measurements on the annealed samples as shown in Figure 2. The present study is not focused on understanding the physical processes underlying the formation of these pop-ins. The goal of this work to characterize the as-received material state (especially in deformed samples) with regard to its ability to initiate bulk crystallographic slip in the indentation zone in situations closer to what would happen when a macroscopic plastic deformation in imposed on the sample (i.e., something akin to the concept of the critical resolved shear stress used in crystal plasticity theories). Therefore, the pop-ins need to be avoided as they obfuscate what we seek to do in this study. Hence, $Y_{\text {ind }}$ was extracted by back-extrapolating from the post-elastic segment shown between the two vertical dashed lines in Figure 2. In other words, this would have been the indentation yield point if there were enough dislocations in the indentation zone to set up potent dislocation sources. In this study, $Y_{\text {ind }}$ was determined as the point of intersection between the modulus line (i.e., the elastic portion of the initial loading segment) and the best-fit line for the stress-strain curve within the strain range of 0.0065 and 0.013 . This protocol was standardized for all the tests reported in this paper. Macroscale deformation increases the dislocation density, and hence, in the deformed samples, the pop-ins are almost completely absent. In order to maintain consistency, the same back-extrapolation method was still used to extract the indentation yield point in the deformed samples as well.

\section{Results and Discussion}

The OIM scans of one of the four samples tested in the fully annealed condition is shown in Figure 3(a). All of the grain orientations tested in the three annealed samples (only one is shown in Figure 3(a)) are plotted in the standard [001] IPF (inverse pole figure) map shown in Figure 3(d). In other words, the color in the OIM scan is mapped to the crystal direction in the grain that is perpendicular to the sample surface (i.e., parallel to the indentation direction). Note that since the indenter tip is axisymmetric, in-plane rotations of the sample (and the corresponding rotations of the crystal orientations with respect to a fixed reference frame) will not alter the nanoindentation response. Consequently, the nanoindentation response is only a function of two of the three Bungle Euler angles used to represent the grain orientation, denoted as $\left(\Phi, \varphi_{2}\right)$.

Representative load-displacement and corresponding ISS curves for two selected grain orientations are plotted in Figure 4. Grain \#1 is close to the [001] corner of the IPF triangle, meaning that the [001] crystal direction for this grain is close to the indentation direction. On the other hand, Grain \#27 is close to the [111] corner and therefore has the [111] crystal direction almost parallel to the indentation direction. As expected, these two orientations show a very large difference in their nanoindentation response. Multiple (9-18) indentation measurements were 
performed on each grain to quantify the experimental scatter in the extracted $Y_{\text {ind }}$ values. A total of twenty seven grain orientations were tested in the annealed samples using the $100 \mu \mathrm{m}$ spherical tip. These grains were selected so that their orientations covered the fundamental stereographic triangle in a uniform manner (see Figure 3(d)). The results from all of these tests are summarized in Table 1. It was observed that the nanoindentation responses within each grain exhibited relatively low deviations in the extracted values of both $E_{\text {ind }}$ and the $Y_{\text {ind }}$ (see Table 1).

As noted earlier, the measured values of $E_{\text {ind }}$ do not exhibit any significant dependence on the grain orientation. However, the measured values of $Y_{\text {ind }}$ exhibit a strong dependence on the grain lattice orientation. In fact, $Y_{\text {ind }}$ can vary by as much as $40 \%$ depending on the orientation of the grain. Figure 5 is a contour plot showing the dependence of the $Y_{\text {ind }}$ on the local crystal lattice orientation at the indentation site in the standard IPF plot. This plot was generated by interpolating between the measured $Y_{\text {ind }}$ values for the 27 grains provided in Table 1 (see also Figure 3(d)). This interpolation was accomplished using generalized spherical harmonics (GSH) [36][ref]. This contour plot indicates that the lowest value of $Y_{\text {ind }}$ would be for a cube-oriented grain $(\sim 40 \mathrm{MPa})$, while the highest value of $Y_{\text {ind }}$ would correspond to a [111] oriented grain $(\sim$ $65 \mathrm{MPa}$ ). Since the dislocation density in an annealed material is expected to be very low and independent of the crystal lattice orientation, all of this variation in the $Y_{\text {ind }}$ with respect to the crystal orientation is fully attributable to the differences in the orientations of the active slip systems with respect to the indentation direction. Since all fcc crystals exhibit the same set of slip systems in plastic deformation at low temperatures (where plastic response is close to a rateindependent response), we should expect the contour plots of $Y_{\text {ind }}$ in the orientation space should look alike for all fcc crystals, except for a constant scaling factor. This hypothesis, however, needs to be verified experimentally.

A similar indentation yield map has been previously reported [19] for annealed grains of $\mathrm{Fe}-3$ $\mathrm{wt} \% \mathrm{Si}$ (a bcc metal). Interestingly, the range in the values of $Y_{\text {ind }}$ is significantly higher in the fcc metal reported here. This can be explained as a natural consequence of the availability of a much larger number of potential slip systems in the bcc crystals, which reduces the plastic anisotropy at the single crystal level. It is also noted that the softest orientations and the hardest orientations for indentation are the same (i.e., [001] and [111] orientations, respectively) in both fcc and bcc metals. The main difference in the $Y_{\text {ind }}$ plots measured in the bcc and fcc crystals appears to be in the relative (i.e., percentage) difference in the $Y_{\text {ind }}$ values for the [111] and [101] orientations. This difference appears to be more significant in the fcc crystals compared to the bcc crystals. However, it is very important to recognize that only a limited number of measurements have been reported to date in both systems, and additional measurements are critically needed to verify and confirm these fairly limited observations.

The yield contour presented in Figure 5 has tremendous value in studies on deformed polycrystalline samples. Using this interpolation plot, $Y_{\text {ind }}$ for any given orientation of pure aluminum in the fully annealed condition can now be estimated. As noted earlier, measurements of $Y_{\text {ind }}$ within the grains in deformed polycrystalline samples are likely to be influenced by both 
the local orientation at the indentation site and the local accumulated dislocation density. It can be argued that the percentage increase in the $Y_{\text {ind }}$ from a reference annealed state for any selected lattice orientation should directly reflect the percentage increase in the local slip resistance (also referred as critical resolved shear strength or CRSS). In this type of analysis, it is implicitly assumed that the slip resistance is the same on all slip systems in the indentation zone. This is a reasonable assumption for cubic polycrystalline metals. We shall now focus on characterizing the local mechanical behavior in the deformed samples using this approach, with the goal of obtaining insights into strain hardening rates in the grains of different orientations in the plastically deformed samples of Al.

As described earlier, two high purity aluminum samples were subjected to light deformation (10\% reduction in height) and another was subjected to moderate deformation (20\% reduction in height); both these reductions were applied in a channel-die to attain plane strain conditions at the sample scale. Representative OIM maps of the sample deformed to $10 \%$ reduction in height and 20\% reduction in height are shown in Figures 3(b) and 3(c), respectively. The orientations of all grains studied at these reduction levels are shown in Figures 3(e) and 3(f), respectively. It is immediately apparent from the OIM maps that there is a much larger spread in the orientations within the grains in the deformed samples. Furthermore, the orientation spread is significantly higher for the sample with higher amount of deformation (20\% reduction in height) as compared to the lightly deformed sample (10\% reduction in height). However, no significant grain fragmentation was observed in either of these samples. All of these observations are consistent with observations reported in earlier studies [38-39]. Indeed, the heterogeneity of both structure and local properties is a characteristic feature of deformed polycrystalline metal samples, and is responsible for important phenomena such as recrystallization [40-41]. One can qualitatively explain this evolving heterogeneity (both between the grains and within the grains) as a natural consequence of the need to accommodate the overall imposed plastic deformation in ways that satisfy the governing field equations (i.e., equilibrium equations) while utilizing only the available slip systems in each grain (in fcc metals there are only twelve (111)[1시 potential slip systems in each grain). However, to develop a more quantitative understanding of the physics controlling the evolution of the microstructure in deformed polycrystalline metals, it would be necessary to develop and employ the novel characterization techniques described in this paper. One of the significant gaps in our current understanding of the grain-scale plasticity comes from a lack of reliable quantitative information on how the individual crystals harden in the process of accommodating the macroscale imposed plastic deformation. In other words, as noted earlier, we expect different grains to undergo different amounts of plastic deformation locally in response to the macroscale imposed plastic deformation (in this study, this was selected as $10 \%$ and $20 \%$ reduction levels in plane strain compression). Consequently, they should harden by different amounts in the process of accommodating the imposed plastic deformation. The novel techniques described in this paper are specifically aimed at measuring the increases in local yield strength in individual grains in a deformed sample and correlating these increases to the crystal orientation. 
Spherical nanoindentation tests were performed on selected regions within the individual grains (well away from visible grain boundaries) using the same protocols that were used on the annealed samples. In this case, the pop-ins were almost always completely absent. The results obtained from the deformed samples are summarized in Tables 2 and 3 for the two different reduction levels. It was once again observed that multiple indentations performed on same grains produced fairly consistent measurements. As noted earlier, at the low reduction levels studied here, the grains have not yet fragmented in any significant manner (see Figures 3(b) and 3(c)). Consequently, the variance in the measurements within a single grain is still relatively low in the measurements reported in this study.

As a specific example of the effect of plastic deformation on $Y_{\text {ind }}$, we show in Figure 6 ISS curves for two grains of almost the same orientation, where one was tested in the fully annealed condition and the other in the $10 \%$ deformed sample. As seen here, the increment in the $Y_{\text {ind }}$ due to the imposed deformation is quite significant. For each of the grains tested in the deformed condition, the $Y_{\text {ind }}$ in the fully annealed condition was estimated using the indentation yield map presented in Figure 5, and the values are presented in Tables 2 and 3. This is the $Y_{\text {ind }}$ value that would have been measured for the orientations studied in the deformed sample, if they were tested in the fully annealed condition. It is important to reliably establish this value since the $Y_{\text {ind }}$ in the annealed condition can vary by as much as $40 \%$, depending on the local crystal lattice orientation at the indentation site. A reliable estimate of the increment in $Y_{\text {ind }}$ (i.e., $\Delta Y_{\text {ind }}$ ) for any given orientation can then be determined as the difference between the measured indentation yield point in the deformed condition $\left(Y_{\text {ind }}(g, d)\right)$ and the estimated indentation yield point in the fully annealed condition, $Y_{\text {ind }}(g, O)$, as

$$
\Delta Y_{\text {ind }}(g, d)=Y_{\text {ind }}(g, d)-Y_{\text {ind }}(g, 0)
$$

where $d$ refers to the amount of deformation (i.e., $10 \%$ or $20 \%$ reduction in height by plane strain compression) and $g$ refers to the local crystal lattice orientation at the indentation site.

The simplest relationship that one can try to establish between $Y_{\text {ind }}$ and the local dislocation content $(\rho)$ is through the critical resolved shear strength $\left(\tau_{\text {crss }}\right)$ of the slip system. In a highly simplified manner, this relationship can be expressed as

$$
\begin{aligned}
& Y_{\text {ind }}(g, d)=M(g) \tau_{\text {crss }}(d) \\
& \Delta \tau_{\text {crss }}(d)=\tau_{\text {crss }}(d)-\tau_{\text {crss }}(0) \propto \sqrt{\rho}
\end{aligned}
$$

where $M$ is a Taylor factor for indentation that depends only on the grain orientation with respect to the indentation direction (in this case, only two of the three Bunge-Euler angles describing local crystal orientation), $\tau_{c r s s}$ is the critical resolved shear strength in the crystal, $\Delta \tau_{\text {crss }}$ is the increment in the local averaged critical resolved shear strength between the annealed and deformed conditions, and $\rho$ is the local dislocation density. The Taylor factor $M$ is defined as 
$M=\frac{\sum \Delta \gamma}{\Delta \varepsilon}$

where $\Delta \gamma$ is the sum of the slip shears on all the slip systems, and $\Delta \varepsilon$ is the imposed macroscopic plastic strain increment. In other words, a grain with a higher value of $M$ is expected to require a higher amount of total slip on its available slip systems to accommodate the imposed macroscopic plastic deformation. Grains with higher values of $M$ are generally referred as hard (oriented) grains, and those with lower values of $M$ are generally referred as soft grains.

Based on the highly simplified crystal plasticity theory presented above for indentation, grains with higher values of $M$ (i.e., hard grains) are expected to produce more amounts of total slip and therefore harden more than the grains with lower values of $M$. This is the fundamental hypothesis we aim to critically evaluate in this study using the novel protocols described here.

A convenient measure for comparing the relative hardening rates in the differently oriented grains in a given deformed sample is to express the increase in the slip resistance as a percentage of the initial critical resolved shear stress in the material in the annealed condition. Combining Eqs. (4)-(6), the percentage increase in the critical resolved shear stress is expressed as

$$
\% \tau_{c r s s}=\frac{\Delta \tau_{c r s s}(d)}{\tau_{c r s s}(0)} * 100=\frac{\Delta Y_{\text {ind }}(g, d)}{Y_{\text {ind }}(g, 0)} * 100
$$

As noted earlier, $\% \tau_{\text {crss }}$ provides an indirect measure of the local dislocation content or local hardening in the deformed samples (cf. Eq. (6)).

From Tables 2 and 3 it is clear that $\% \tau_{\text {crss }}$ (reflecting the local hardening rates) does vary significantly with the grain orientation. Also reported in the Tables 2 and 3 are values of the Taylor factor for plane strain compression for each of the orientation of interest. The definition of the Taylor factor provided in Eq. (7) is general and can be applied to any imposed deformation mode. The values presented in Tables 2 and 3 are for plane strain compression, and are not to be confused with the Taylor factors for the indentation mode of deformation used in Eq. (5). Taylor factors shown in Tables 2 and 3 indicate how the specific orientations will accommodate an imposed plane strain compression (there is an implicit assumption here that the grain will experience locally the same deformation as the macroscale imposed deformation; this assumption is usually referred to as the Taylor assumption [42]). As noted earlier, our main hypotheses from the simple model presented earlier is that the grains with higher Taylor factors for plane strain compression are expected to require a larger amount of total slip, and consequently harden more than the grains with lower Taylor factors.

Figures $7(\mathrm{a})$ and $7(\mathrm{~b})$ show the variation of $\% \tau_{\text {crss }}$ (estimated from the nanoindentation measurements conducted in this study) with the Taylor factors for all grain orientations studied at both reduction levels (i.e., $10 \%$ and $20 \%$ reduction by plane strain compression), respectively. It is seen that there is a strong positive correlations between the local strain hardening levels in the 
individual grains and the Taylor factors computed for their lattice orientations. In fact, in both sets of measurements shown in Figures 7(a) and 7(b), there is a strong suggestion for a linear relationship between $\% \tau_{c r s s}$ and $M$.

The positive correlations between $\% \tau_{c r s s}$ and $M$ is a very important finding and supports the main hypothesis laid out earlier. The data presented here confirms that orientations with a lower Taylor factor (soft grains) harden at a lower rate compared to grains with a higher Taylor factor (hard grains). This dependence has been discussed in various books and has been probed previously within a limited scope [43-44]. To the best of our knowledge, the dataset presented in this paper represents the most comprehensive set of measurements conducted to date in support of this expected correlation.

Interestingly enough, and as noted earlier, Figures 7(a) and 7(b) actually suggest a linear relationship between $\% \tau_{c r s s}$ and $M$. The observed linear dependence can be reconciled using a highly simplified model for the hardening rate in each grain. If we assume that the initial hardening rate in the differently oriented crystals in their annealed condition is constant (denoted by $h$ ) and relatively independent of the grain orientation, we can express the increase in slip resistance as

$$
\Delta \tau_{c r s s}=h \sum \Delta \gamma
$$

Combining Eq. (9) with Eq. (7) we can show that

$$
\% \tau_{c r s s}=M\left(\frac{h^{*} \Delta \varepsilon}{\tau_{c r s s}(0)}\right)
$$

Eq. (10) is consistent with the linear correlations observed in Figures 7(a) and 7(b).

The simple model presented above, however, assumes that the initial hardening rate (i.e., the stage II hardening rate) in the individual crystals is constant for all grain orientations. It is indeed remarkable that very simple models discussed above correctly predict the main trends in the measurements obtained in this study. It is also anticipated that the measurement dataset produced in this study would be of high value to experts engaged in the development of refined crystal plasticity theories and simulation tools. We have therefore presented all of the relevant details of the measurements in this paper (see Tables 1-3), so that the broader scientific community can re-analyze this dataset using their own favorite models, and draw their own conclusions and correlations.

From Tables 1-3, it is also seen that the scatter in the measured $Y_{\text {ind }}$ values for individual grains is higher for the deformed samples as compared to the scatter observed for the annealed samples. This is expected because, for the deformed samples, in addition to the experimental scatter in the $Y_{\text {ind }}$ values, there is also a real variation in the dislocation density from one location to another within individual grains which contributes to the scatter. 


\section{Conclusions}

A new set of protocols have been developed to reliably measure the local percentage increases in slip resistance (from their values in the annealed condition) in individual grains of a deformed cubic polycrystal sample. In the present study, the protocols were specifically demonstrated for Al polycrystals. However, we believe that the same set of protocols can be applied to a range of cubic metals deforming by crystallographic slip. The overall approach combines the use of spherical nanoindentation stress-strain curves with electron-backscattered diffraction for orientation imaging. The proposed protocol is essentially a two-step process. In the first step, the dependence of the indentation yield strength $\left(Y_{\text {ind }}\right)$ on the crystal lattice orientation is mapped out in the fully annealed condition. In the second step, this function is utilized to carefully isolate the contribution from the increase in the critical resolved shear strength (due to increased dislocation density) to the $Y_{\text {ind }}$ measured in deformed samples. The protocols developed here were applied to deformed samples of polycrystalline $\mathrm{Al}$ and the results showed a remarkable agreement with a highly simplified theory. These new protocols have the potential to open several new lines of investigations in fundamental studies of deformation and recrystallization in polycrystalline metals.

\section{Acknowledgements}

Authors wish to thank Prof. R. D. Doherty for the many insightful discussions during the course of this work. Authors acknowledge funding from ARO grant W911NF-10-1-0409 (David Stepp, Program Manager). The MTS nanoindenter XP® and the TSL-OIM system (integrated with the ESEM Philips XL-30) used in this study is maintained and operated by the Centralized Research Facilities in the College of Engineering at Drexel University. SJV is currently at Los Alamos National Laboratory, operated by LANS for the NNSA of the US Department of Energy under Contract No. DE-AC52-06NA25396.

\section{References}

1. Hay, J.L. and G.M. Pharr, Instrumented indentation testing, ASM Handbook.

2. Hay, J.C., A. Bolshakov, and G.M. Pharr, A critical examination of the fundamental relations used in the analysis of nanoindentation data. Journal of Materials Research, 1999. 14(6): p. 22962305.

3. Fischer-Cripps, A.C., Nanoindentation. Mechanical Engineering Series, ed. F.F. Ling. 2002, New York: Springer.

4. Fischer-Cripps, A.C., A review of analysis methods for sub-micron indentation testing. Vacuum, 2000. 58(4): p. 569-585.

5. Uchic, M.D., et al., Sample dimensions influence strength and crystal plasticity. Science, 2004. 305(5686): p. 986-989.

6. Shan, Z.W., et al., Mechanical annealing and source-limited deformation in submicrometrediameter Ni crystals. Nat Mater, 2008. 7(2): p. 115-119.

7. Kunz, A., S. Pathak, and J.R. Greer, Size effects in Al nanopillars: Single crystalline vs. bicrystalline. Acta Materialia, 2011. 59(11): p. 4416-4424.

8. Bucaille, J.L., et al., Determination of plastic properties of metals by instrumented indentation using different sharp indenters. Acta Materialia, 2003. 51(6): p. 1663-1678. 
9. Rho, J.-Y., T.Y. Tsui, and G.M. Pharr, Elastic properties of human cortical and trabecular lamellar bone measured by nanoindentation. Biomaterials, 1997. 18(20): p. 1325-1330.

10. Poole, W.J., M.F. Ashby, and N.A. Fleck, Micro-hardness of annealed and work-hardened copper polycrystals. Scripta Materialia, 1996. 34(4): p. 559-564.

11. Oliver, W.C. and G.M. Pharr, Improved technique for determining hardness and elastic modulus using load and displacement sensing indentation experiments. Journal of Materials Research, 1992. 7(6): p. 1564-1580.

12. Oliver, W.C. and G.M. Pharr, Measurement of hardness and elastic modulus by instrumented indentation: Advances in understanding and refinements to methodology. Journal of Materials Research, 2004. 19: p. 3-20.

13. Fischer-Cripps, A.C., Study of analysis methods of depth-sensing indentation test data for spherical indenters. Journal of Materials Research, 2001. 16: p. 1579-84.

14. $\mathrm{Li}, \mathrm{X}$. and $\mathrm{B}$. Bhushan, A review of nanoindentation continuous stiffness measurement technique and its applications. Materials Characterization, 2002. 48(1): p. 11-36.

15. Kalidindi, S.R. and S. Pathak, Determination of the effective zero-point and the extraction of spherical nanoindentation stress-strain curves. Acta Materialia, 2008. 56: p. 3523-32.

16. Pathak, S., et al., Studying grain boundary regions in polycrystalline materials using spherical nano-indentation and orientation imaging microscopy. Journal of Materials Science, 2012. 47(2): p. 815-823.

17. Pathak, S., et al., Viscoelasticity and high buckling stress of dense carbon nanotube brushes. Carbon, 2009. 47: p. 1969-1976.

18. Pathak, S., et al., Assessment of lamellar level properties in mouse bone utilizing a novel spherical nanoindentation data analysis method. Journal of the Mechanical Behavior of Biomedical Materials, 2012. 13(0): p. 102-117.

19. Pathak, S., D. Stojakovic, and S.R. Kalidindi, Measurement of the local mechanical properties in polycrystalline samples using spherical nanoindentation and orientation imaging microscopy. Acta Materialia, 2009. 57: p. 3020-8.

20. Adams, B.L. Measurement and representation of polycrystalline microstructure. in Winter Annual Meeting of the American Society of Mechanical Engineers, Nov 8-13 1992. 1992. Anaheim, CA, USA: Publ by ASME, New York, NY, USA.

21. Adams, B.L., Orientation imaging microscopy: Emerging and future applications. Ultramicroscopy Proceedings of the 1996 6th Conference on Frontiers in Electron Microscopy in Materials Science, Jun 4-7 1996, 1997. 67(1-4): p. 11-17.

22. Asaro, R.J., Crystal Plasticity. Journal of Applied Mechanics, 1983. 50(4b): p. 921-934.

23. Bronkhorst, C.A., S.R. Kalidindi, and L. Anand, Polycrystalline plasticity and the evolution of crystallographic texture in FCC metals. Philosophical Transactions of the Royal Society of London Series A-Mathematical Physical and Engineering Sciences, 1992. 341(1662): p. 443-477.

24. Kalidindi, S.R., Incorporation of deformation twinning in crystal plasticity models. Journal of the Mechanics and Physics of Solids, 1998. 46(2): p. 267-271.

25. Van Houtte, P., L. Delannay, and S.R. Kalidindi, Comparison of two grain interaction models for polycrystal plasticity and deformation texture prediction. International Journal of Plasticity, 2002. 18(3): p. 359-377.

26. Pathak, S., J. Shaffer, and S.R. Kalidindi, Determination of an effective zero-point and extraction of indentation stress-strain curves without the continuous stiffness measurement signal. Scripta Materialia, 2009. 60: p. 439-42.

27. Hertz, H., Miscellaneous Papers. New York: MacMillan and Co., Ltd, 1896.

28. Johnson, K.L., Indentation Contact Mechanics. 1985: Cambridge University Press, Cambridge.

29. Donohue, B.R., A. Ambrus, and S.R. Kalidindi, Critical evaluation of the indentation data analyses methods for the extraction of isotropic uniaxial mechanical properties using finite element models. Acta Materialia, 2012. 60(9): p. 3943-3952. 
30. G.M. Pharr, J.H.S.a.W.C.O., Critical issues in making small-depth mechanical property measurements by nanoindentation with continuous stiffness measurement. Journal of Materials Research, 2009. 24: p. 653-666.

31. Vachhani, S.J., R.D. Doherty, and S.R. Kalidindi, Effect of the continuous stiffness measurement on the mechanical properties extracted using spherical nanoindentation. Acta Materialia, 2013. 61(10): p. 3744-3751.

32. Gene Simmons, H.W., Single Crystal Elastic Constants and Calculated Aggregate Properties. 1971, Cambridge MIT Press.

33. Vlassak, J.J. and W.D. Nix, Measuring the elastic properties of anisotropic materials by means of indentation experiments. Journal of the Mechanics and Physics of Solids, 1994. 42(8): p. 12231245.

34. Metallography and Microstructures, in ASM handbooks Online, G.F.V. Voort, Editor. 2004.

35. Pathak, S., et al., Importance of surface preparation on the nano-indentation stress-strain curves measured in metals. Journal of Materials Research, 2009. 24: p. 1142-55.

36. Bunge, H., Texture Analysis in Materials Science. Butterworths, 1982.

37. Pathak, S., et al., Understanding pop-ins in spherical nanoindentation. Applied Physics Letters, 2014. 105(16): p. 161913.

38. Kalidindi, S.R., A. Bhattacharya, and R. Doherty, Detailed Analysis of Plastic Deformation in Columnar Polycrystalline Aluminum Using Orientation Image Mapping and Crystal Plasticity Models. Proceedings of the Royal Society of London: Mathematical, Physical and Engineering Sciences., 2004. 460(2047)): p. 1935 - 1956

39. Bhattacharyya, A., et al., Evolution of grain-scale microstructure during large strain simple compression of polycrystalline aluminum with quasi-columnar grains: OIM measurements and numerical simulations. International Journal of Plasticity, 2001. 17(6): p. 861-883.

40. Humphreys, F.J. and M. Hatherly, Recrystallization and Related Annealing Phenomena. $2^{\text {nd }}$ ed. 2004, Amsterdam: Elsevier.

41. Doherty, R.D., et al., Current issues in recrystallization: a review. Materials Science \& Engineering A, 1997. 238: p. 214-274.

42. Taylor, G.I., Plastic strain in metals. Journal of the Institute of Metals, 1938. 62: p. 307-324.

43. Dillamore, I.L., Morris P. L., Smith C. J. E. , Hutchinson W. B., Transition Bands and Recrystallization in Metals. Proceedings of the Royal Society A, 1972. 329: p. 405-420.

44. Stojakovic, D., Micorstructure Evolution in Deformed and Recrystallized Electrical Steel, in Materials Science and Engineering. 2008, Drexel University: Philadlephia. p. 101. 


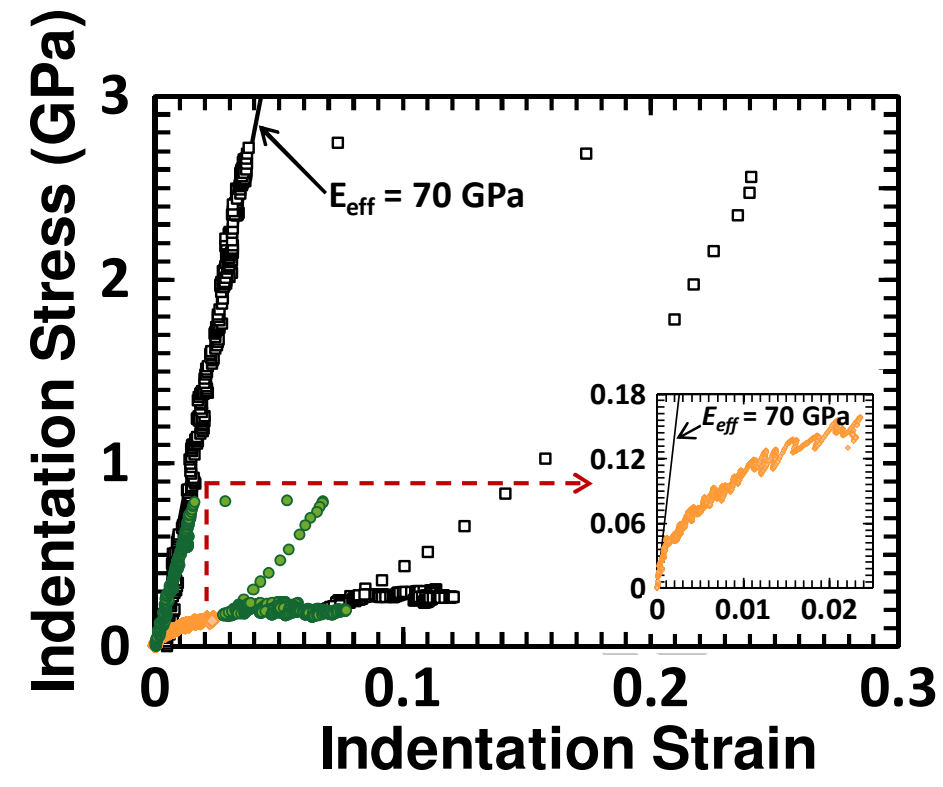

$\checkmark 1 \mu \mathrm{m}$ tip $\odot 5 \mu \mathrm{m}$ tip $\diamond 100 \mu \mathrm{m}$ tip

Figure 1. Representative ISS curves for tests performed within the same grain in the fully annealed aluminum sample using the 1,5 and $100 \mu \mathrm{m}$ indenter tips. Pop-ins mask the conventional elastic-plastictransition and make the determination of the $Y_{\text {ind }}$ difficult. The size of the pop-in reduces rapidly with an increase in the indenter tip size and is absent for the test with the $100 \mu \mathrm{m}$ indenter tip (expanded in the inset). 


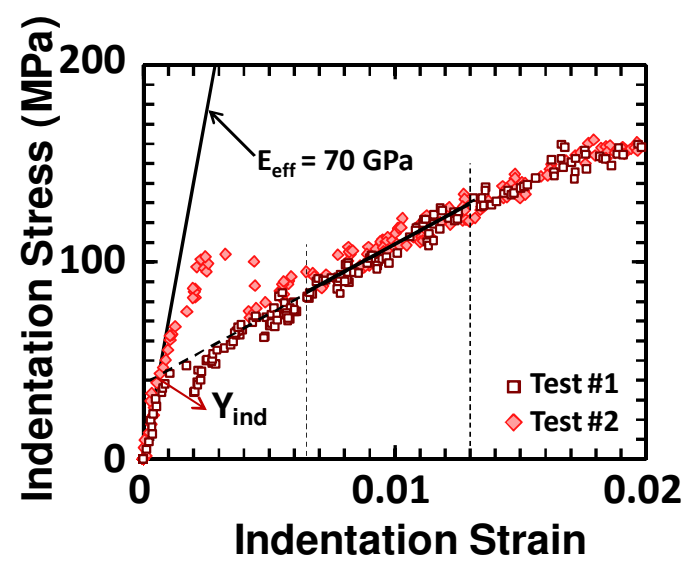

Figure 2: ISS curves for two tests carried out within the same grain in the fully annealed aluminum sample using the $100 \mu \mathrm{m}$ indenter tip. As seen here, the pop-ins are occasionally observed but are small enough that the back-extrapolation method can be reliably used to extract $Y_{\text {ind }}$ values. The dashed vertical lines show the segment of the stress-strain curve used for the back extrapolation. This segment is carefully selected to be just outside the pop-in segment. 


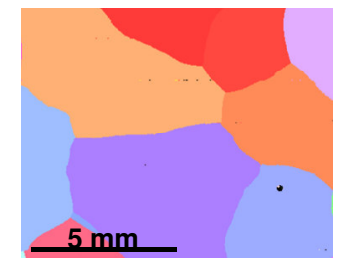

(a)

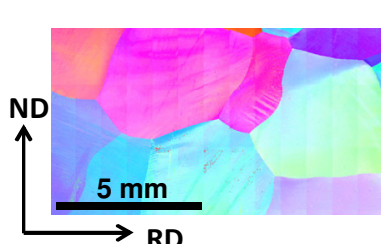

(b)

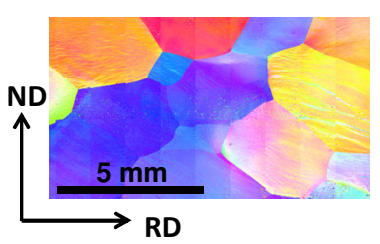

(c)

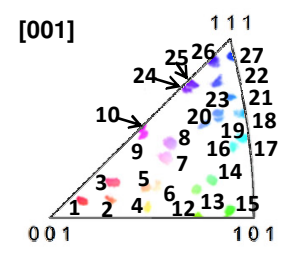

(d)

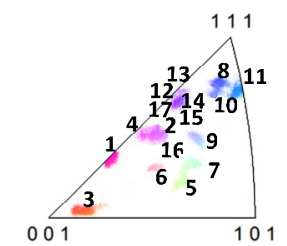

(e)

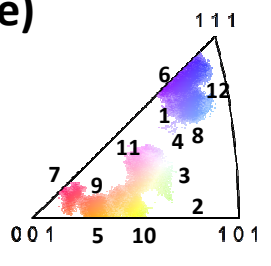

(f)

Figure 3: Representative OIM maps from the pure aluminum samples studied in the (a) fully annealed condition, (b) after $10 \%$ and (c) $20 \%$ reduction in height by plane strain compression. The IPF maps showing the positions of all the grains tested in the (d) fully annealed condition, after (e) $10 \%$ and (f) $20 \%$ reduction in height respectively. Since the grains are very large ( $2-3 \mathrm{~mm}$ ) only a limited number of orientations are present in each sample and multiple samples were employed to gather adequate amount of data for the study. 


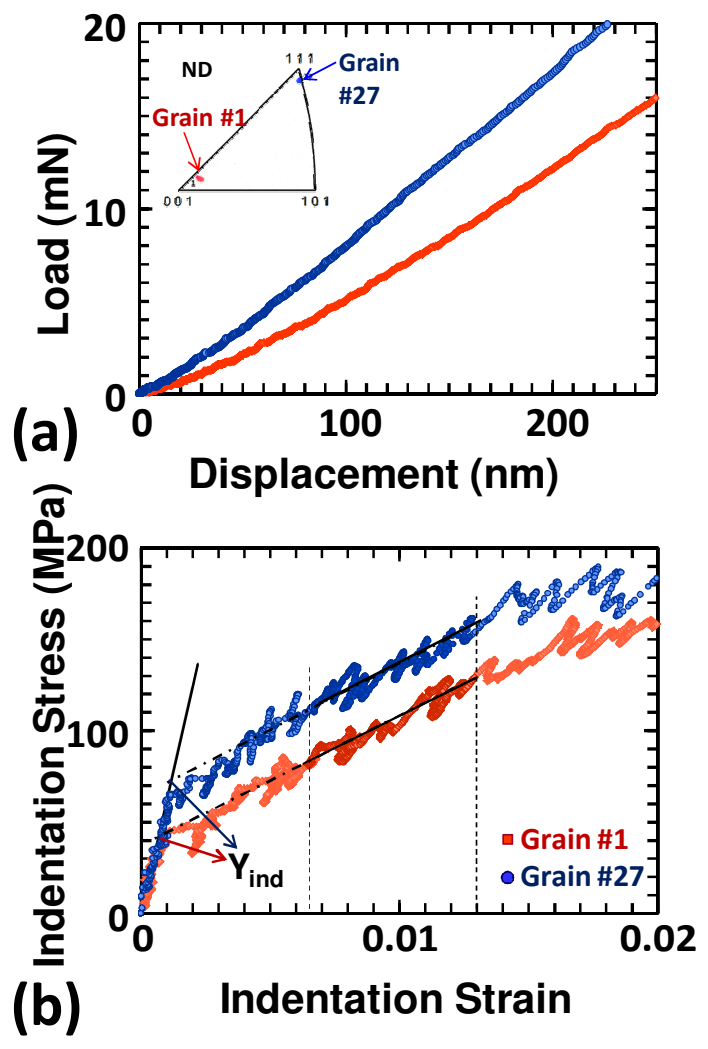

Figure 4: The measured load-displacement data for two grains (grain \#1 and grain \#27) in the fully annealed condition. The inset shows the IPF map which indicates the orientation of the grains with respect to the indentation axis. The corresponding ISS curves are in (b). Note that the $Y_{\text {ind }}$ values vary quite significantly between the two orientations. 


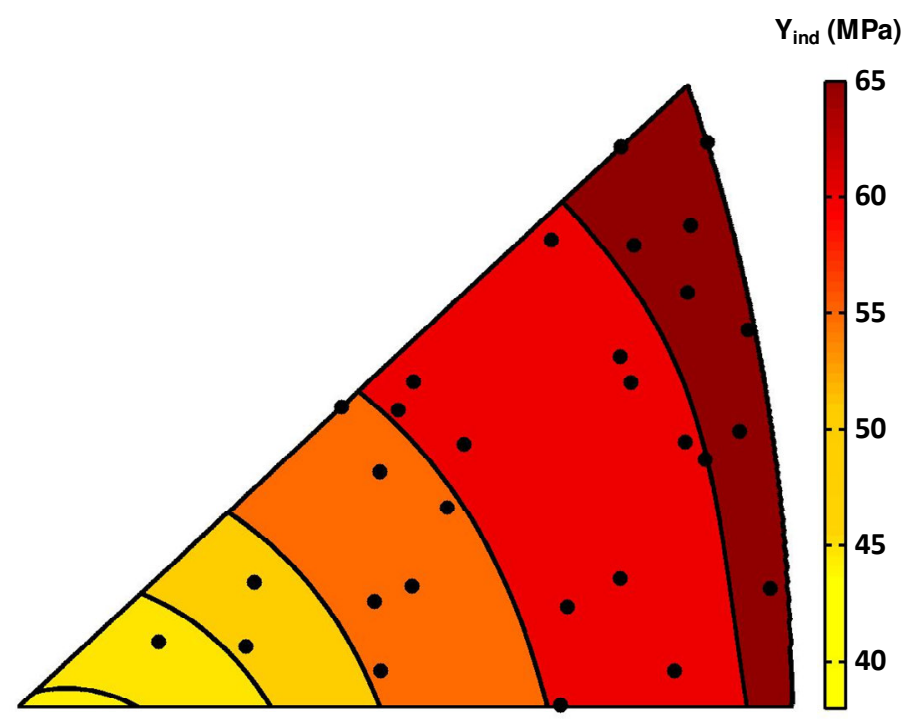

Figure 5: Contour plot generated by interpolating between the average $Y_{\text {ind }}$ values extracted for the 27 grains in the annealed condition. The black circles represent the positions of the tested grains on the standard IPF plot. 


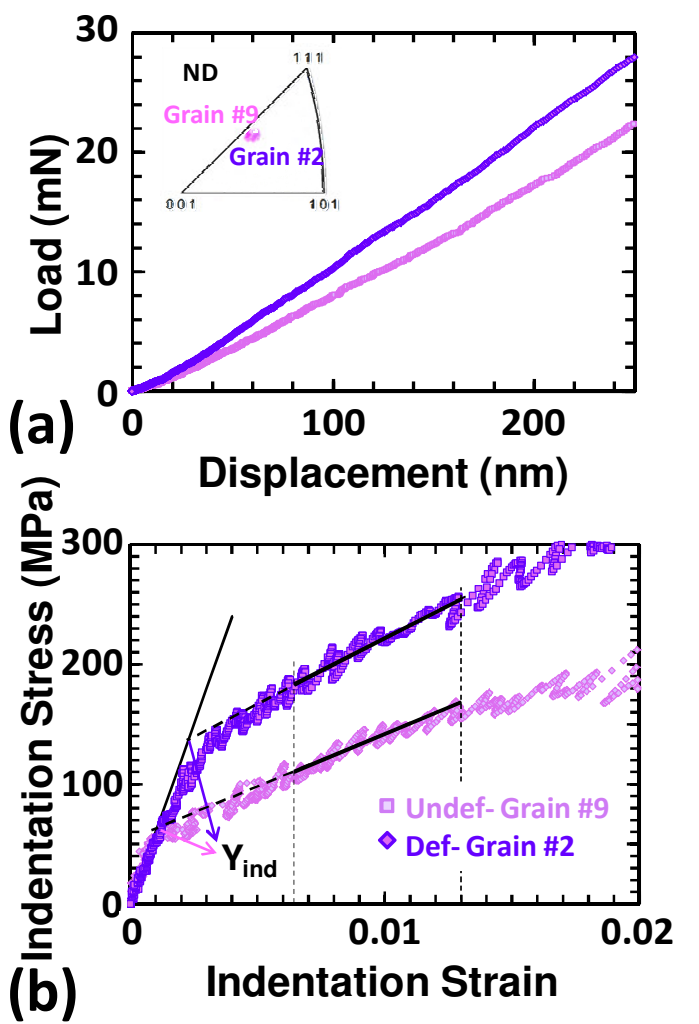

Figure 6: (a) The load-displacement data, and (b) corresponding indentation stress-strain curves, for two almost identically oriented grains ( $4.3^{\circ}$ misorientation), showing the effect of deformation on the ISS response. Grain \#9 was tested in the fully annealed condition and Grain \#2 was tested after the sample was deformed to $10 \%$ reduction in height by plane strain compression. 

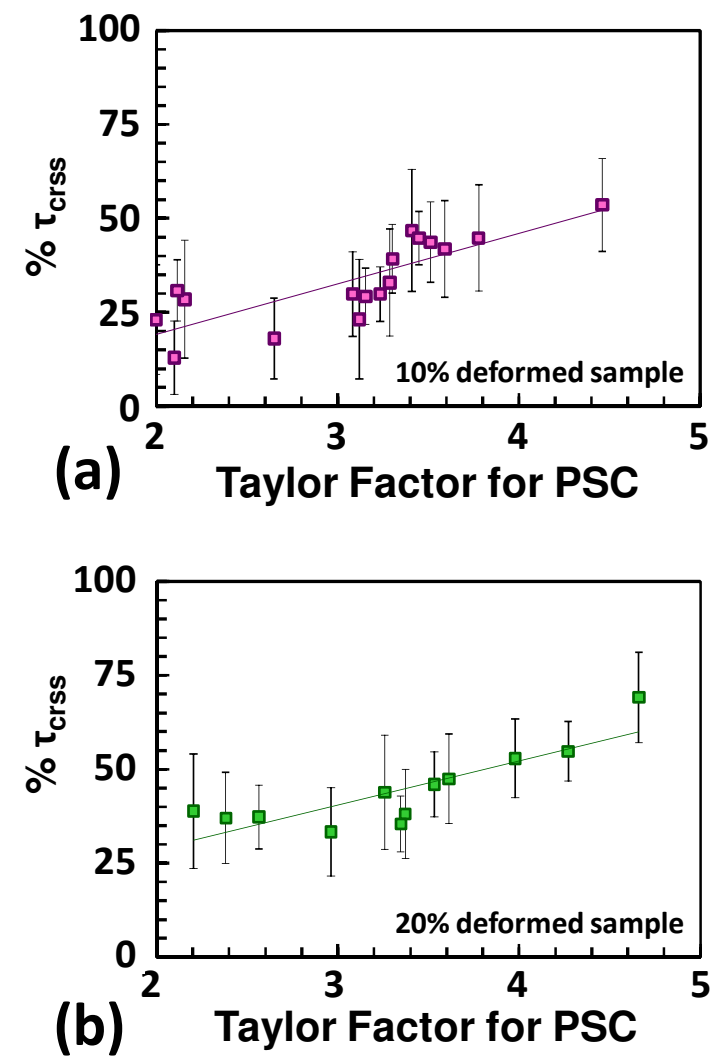

Figure 7: Plot of the percentage increase in the measured critical resolved shear stress, for individual grains, as a function of the Taylor factor in plane strain compression computed for the grains. (a) $10 \%$ reduction level, and (b) 20\% reduction level. 


\begin{tabular}{|c|c|c|c|c|c|}
\hline \multirow{2}{*}{ Grain No. } & \multicolumn{3}{|c|}{ Orientation (Euler Angles) } & \multirow{2}{*}{$E_{\text {ind }}(\mathbf{G P a})$} & \multirow{2}{*}{$\mathbf{Y}_{\text {ind }}(\mathbf{M P a})$} \\
\hline & $\varphi_{1}$ & $\Phi$ & $\varphi_{2}$ & & \\
\hline 1 & 73.1 & 9.8 & 63.4 & $74.3 \pm 0.9$ & $49.3 \pm 5$ \\
\hline 2 & 224.9 & 14.7 & 73.9 & $75.0 \pm 1.0$ & $53.6 \pm 3$ \\
\hline 3 & 219.9 & 16.8 & 60.3 & $75.8 \pm 0.3$ & $50.1 \pm 8$ \\
\hline 4 & 143 & 22.4 & 83.7 & $75.4 \pm 0.2$ & $60.7 \pm 5$ \\
\hline 5 & 347.9 & 23 & 72.2 & $74.8 \pm 1.0$ & $60.8 \pm 1$ \\
\hline 6 & 29 & 25.4 & 71.6 & $74.8 \pm 1.9$ & $54.1 \pm 4$ \\
\hline 7 & 60.9 & 29.2 & 63.4 & $75.1 \pm 1.0$ & $60.8 \pm 8$ \\
\hline 8 & 348.5 & 32 & 57.6 & $75.1 \pm 1.1$ & $65.1 \pm 1$ \\
\hline 9 & 190.1 & 30.2 & 49.9 & $75.1 \pm 0.5$ & $65.1 \pm 9$ \\
\hline 10 & 347.2 & 27.9 & 45 & $74.2 \pm 0.5$ & $60.5 \pm 8$ \\
\hline 11 & 280.5 & 32 & 48.5 & $74.2 \pm 0.3$ & $60.0 \pm 8$ \\
\hline 12 & 23 & 32.8 & 89.7 & $75.1 \pm 0.1$ & $58.1 \pm 41$ \\
\hline 13 & 13.7 & 33.8 & 78.8 & $74.3 \pm 2.5$ & $59.4 \pm 6$ \\
\hline 14 & 306.5 & 37.1 & 77 & $74.1 \pm 0.2$ & $61.9 \pm 7$ \\
\hline 15 & 299.7 & 39.3 & 86.5 & $75.1 \pm 0.9$ & $63.0 \pm 5$ \\
\hline 16 & 273.4 & 43.6 & 68.8 & $75.3 \pm 1.1$ & $65.8 \pm 5$ \\
\hline 17 & 167.3 & 45.9 & 67.6 & $74.3 \pm 0.7$ & $61.9 \pm 1$ \\
\hline 18 & 182.5 & 48.8 & 60.9 & $74.9 \pm 0.5$ & $65.8 \pm 8$ \\
\hline 19 & 232.2 & 42.2 & 57.9 & $75.4 \pm 1.1$ & $64.5 \pm 8$ \\
\hline 20 & 15 & 41.9 & 60.3 & $75.6 \pm 1.2$ & $60.5 \pm 5$ \\
\hline 21 & 207.7 & 47.2 & 56.3 & $74.6 \pm 1.1$ & $65.9 \pm 7$ \\
\hline 22 & 187.6 & 49.5 & 52.4 & $75.2 \pm 0.1$ & $62.2 \pm 4$ \\
\hline 23 & 185.6 & 46.5 & 51.1 & $74.7 \pm 1.8$ & $69.7 \pm 8$ \\
\hline 24 & 288.6 & 43 & 66.8 & $74.8 \pm 0.1$ & $61.8 \pm 7$ \\
\hline 25 & 241.3 & 43.4 & 46.7 & $75.5 \pm 1.5$ & $61.8 \pm 3$ \\
\hline 26 & 45 & 49.7 & 45 & $74.7 \pm 1.0$ & $69.7 \pm 1$ \\
\hline 27 & 45.4 & 53 & 48.6 & $74.6 \pm 1.8$ & $65.5 \pm 5$ \\
\hline
\end{tabular}

Table 5.1: Summary of the measurements on the 27 grains tested in the fully annealed condition. Orientation of the grains are presented as Bunge-Euler angles. The indentation modulus $\left(\mathrm{E}_{\text {ind }}\right)$ and the indentation yield strength $\left(\mathrm{Y}_{\text {ind }}\right)$ were extracted from nanoindentation tests carried out using the $100 \mu \mathrm{m}$ spherical tip. 


\begin{tabular}{|c|c|c|c|c|c|c|c|}
\hline \multirow{2}{*}{ Grain No } & \multicolumn{3}{|c|}{ Orientation (Euler Angles) } & \multirow{2}{*}{$\begin{array}{c}\text { Taylor } \\
\text { Factor for } \\
\text { PSC } \\
\end{array}$} & \multirow{2}{*}{$\begin{array}{c}\text { Predicted } \\
\mathbf{Y}_{\text {ind }} \text { (undef) }\end{array}$} & \multirow{2}{*}{ Yind (MPa) } & \multirow{2}{*}{$\% \tau_{\text {crss }}$} \\
\hline & $\varphi_{1}$ & $\Phi$ & $\varphi_{2}$ & & & & \\
\hline 1 & 180.8 & 19.5 & 45.0 & 4.46 & 54.8 & $85.5 \pm 6.9$ & $53.55 \pm 12.33$ \\
\hline 2 & 328.1 & 31.0 & 52.8 & 3.12 & 61.1 & $74.4 \pm 9.8$ & $23.16 \pm 15.96$ \\
\hline 3 & 257.6 & 9.7 & 80.0 & 3.41 & 46.3 & $68.9 \pm 7.6$ & $46.81 \pm 16.22$ \\
\hline 4 & 43.3 & 33.4 & 51.8 & 2.16 & 62.2 & $78.6 \pm 9.6$ & $28.47 \pm 15.67$ \\
\hline 5 & 44.3 & 30.9 & 72.6 & 3.45 & 60.5 & $87.0 \pm 4.3$ & $44.76 \pm 7.10$ \\
\hline 6 & 58.0 & 25.9 & 63.4 & 3.51 & 58.0 & $83.9 \pm 6.2$ & $43.66 \pm 10.96$ \\
\hline 7 & 62.2 & 32.4 & 68.2 & 3.59 & 61.5 & $86.1 \pm 7.8$ & $41.85 \pm 12.87$ \\
\hline 8 & 251.8 & 47.1 & 51.8 & 3.08 & 66.3 & $85.2 \pm 7.4$ & $29.85 \pm 11.26$ \\
\hline 9 & 97.8 & 38.2 & 66.0 & 2.65 & 64.3 & $74.3 \pm 6.8$ & $17.99 \pm 11.75$ \\
\hline 10 & 155.2 & 44.5 & 56.3 & 3.30 & 66.1 & $87.3 \pm 5.7$ & $39.23 \pm 9.14$ \\
\hline 11 & 256.8 & 49.7 & 53.6 & 3.29 & 66.5 & $86.3 \pm 9.3$ & $32.29 \pm 14.26$ \\
\hline 12 & 129.1 & 38.9 & 47.5 & 2.10 & 064.4 & $74.8 \pm 6.5$ & $12.89 \pm 9.75$ \\
\hline 13 & 270.0 & 35.3 & 45.0 & 3.78 & 63.1 & $89.6 \pm 8.8$ & $44.77 \pm 14.15$ \\
\hline 14 & 36.0 & 42.7 & 49.4 & 2.12 & 65.5 & $84.0 \pm 5.2$ & $30.84 \pm 8.14$ \\
\hline 15 & 135.7 & 38.8 & 48.5 & 2.00 & 64.3 & $82.6 \pm 9.2$ & $23.05 \pm 114.59$ \\
\hline 16 & 274.8 & 29.1 & 54.5 & 3.15 & 60.1 & $77.3 \pm 4.5$ & $29.24 \pm 7.46$ \\
\hline 17 & 231.8 & 36.5 & 53.1 & 3.23 & 63.5 & $80.8 \pm 4.5$ & $29.82 \pm 7.23$ \\
\hline
\end{tabular}

Table 2: The grain orientations, corresponding Taylor factor for PSC, the predicted $Y_{\text {ind }}$ values in the fully annealed condition for the orientations, $Y_{\text {ind }}$ in the deformed condition, measured using indentation, and percentage increase in the critical resolved shear stress, as a result of the imposed macroscopic strain for the sample deformed to $10 \%$ reduction in height by plane strain compression. 


\begin{tabular}{|c|c|c|c|c|c|c|c|}
\hline \multirow{2}{*}{ Grain No } & \multicolumn{3}{|c|}{ Orientation (Euler Angles) } & \multirow{2}{*}{$\begin{array}{c}\text { Taylor } \\
\text { Factor for } \\
\text { PSC }\end{array}$} & \multirow{2}{*}{$\begin{array}{c}\text { Predicted } \\
\mathbf{Y}_{\text {ind }} \text { (undef) }\end{array}$} & \multirow{2}{*}{$\mathbf{Y}_{\text {ind }}(\mathbf{M P a})$} & \multirow{2}{*}{$\% \tau_{\text {crss }}$} \\
\hline & $\varphi_{1}$ & $\Phi$ & $\varphi_{2}$ & & & & \\
\hline 1 & 119 & 43.2 & 56.3 & 2.20 & 64.4 & $90.4 \pm 10.0$ & $38.84 \pm 15.26$ \\
\hline 2 & 13.6 & 38.4 & 77.3 & 2.38 & 62.7 & $88.1 \pm 9.9$ & $37.03 \pm 12.1$ \\
\hline 3 & 143.7 & 28.1 & 69.4 & 3.53 & 59.2 & $86.1 \pm 5.1$ & $46.0 \pm 8.67$ \\
\hline 4 & 189.2 & 35.3 & 48.8 & 3.26 & 61.8 & $90.5 \pm 9.9$ & $43.87 \pm 15.2$ \\
\hline 5 & 152 & 15.8 & 71.2 & 4.66 & 51.9 & $86.1 \pm 7.4$ & $69.09 \pm 11.99$ \\
\hline 6 & 116.8 & 40.5 & 47 & 2.96 & 63.5 & $85.4 \pm 7.9$ & $33.33 \pm 11.77$ \\
\hline 7 & 224.4 & 10.8 & 69.4 & 3.37 & 48.4 & $66.3 \pm 5.7$ & $38.08 \pm 11.87$ \\
\hline 8 & 354 & 43.2 & 59.5 & 3.35 & 64.5 & $86.7 \pm 4.7$ & $35.42 \pm 7.39$ \\
\hline 9 & 250 & 19.6 & 69.4 & 4.27 & 54.7 & $84.4 \pm 4.8$ & $54.73 \pm 7.92$ \\
\hline 10 & 223.8 & 21.9 & 85.2 & 3.98 & 55.2 & $84.1 \pm 5.8$ & $52.88 \pm 10.47$ \\
\hline 11 & 347.5 & 28.4 & 67.4 & 3.61 & 59.3 & $87.0 \pm 7.0$ & $47.46 \pm 11.92$ \\
\hline 12 & 51.4 & 44.8 & 49 & 2.57 & 64.9 & $89.2 \pm 5.5$ & $37.26 \pm 8.49$ \\
\hline
\end{tabular}

Table 3: The grain orientations, corresponding Taylor factor for PSC, the predicted $Y_{\text {ind }}$ values for the orientation in the fully annealed condition, $Y_{\text {ind }}$ measured in the as deformed condition and percentage increase in the critical resolved shear stress, as a result of the imposed macroscopic strain for the sample deformed to $20 \%$ reduction in height by plane strain compression. 